\title{
CARMEN MARTÍN GAITE COMO MEDIADORA EDITORIAL: EL COMPROMISO ARTÍSTICO
}

JOSÉ TERUEL

Universidad Autónoma de Madrid

Este artículo desarrolla el papel de mediación editorial ejercido por Carmen Martín Gaite a través de cinco hitos: su intercesión ante José Vergés para la publicación de la primera novela de Juan Benet (1967), su madrinazgo de la editorial Nostromo (especialmente en los primeros años, 19731974), su breve empleo en Salvat Editores (octubre de 1973-junio de 1974), su vinculación con el comité de lectura para autores extranjeros de Alfaguara (1978), y su papel de cónsul de Anagrama en Madrid (1992-2000). De este itinerario de mediación - escasamente atendido en su biografía intelectual- se desprenden su mecenazgo de los narradores noveles y su compromiso con los valores literarios sobre los empresariales; aunque esta defensa de los jóvenes y este compromiso con lo artístico fueron también un modo de potenciar su auctoritas.

PALABRAS CLAVE: Carmen Martín Gaite, mediación editorial, auctoritas.

\section{Carmen Martín Gaite as Mediator in the Publishing World: Her Artistic Commitment}

This article considers five examples of Carmen Martin Gaite's activity as mediator in the editorial world: her role in convincing José Vergés to publish Juan Benet's first novel (1967), her mentoring work for Nostromo (especially in the early years 1973-74), her brief employment with Salvat Editores (Oct. 1973-June 1974), her association with Alfaguara's committee of readers for foreign authors (1978), and her role as adviser for Anagrama in Madrid (1992-2002). This overview of her role as mediator - practically ignored in her intellectual biographies - allows us to better understand her patronage of young writers and her commitment to giving priority to literary value over commercial interests; although this defense of young writers and this commitment to artistic quality were also ways to underscore her own auctoritas.

KEY WORDS: Carmen Martín Gaite, mediation in the publishing world, auctoritas.

Una cuestión escasamente atendida en la biografía intelectual de Carmen Martín Gaite es su actividad de mediación editorial. Solo en los últimos años con las crónicas de Jorge Herralde, en Opiniones mohicanas (2001) y Por orden alfabético (2006), hemos comenzado a tener noticia de esta faceta de la escritora. A pesar de esa falta de atención, he conseguido recopilar otros testimonios que acreditan su papel de asesoramiento, madrinazgo e incluso empleada editorial y que anteceden su espontánea labor de cónsul de Anagrama en Madrid.

Teruel, José (2019), "Carmen Martín Gaite como mediadora editorial: El compromiso artístico", Lectora, 25: 187-196. ISSN: 1136-5781 D.O.I.: 10.1344/Lectora2019.25.11, jose.teruel@uam.es Recepció: 15 de desembre de 2018 - Acceptació: 15 d'abril de 2019 
El primer documento procede de su Correspondencia con Juan Benet. En una carta de febrero de 1967, dirigida a José Vergés, entonces director de Ediciones Destino, Carmen Martín Gaite aboga por la pronta publicación de Volverás a Región (1968), recalcando la iniciativa que en el mismo sentido ya había emprendido Dionisio Ridruejo: ${ }^{1}$

Me he enterado de que parece usted inclinado a publicar la novela de Juan Benet Volverás a Región. Me parece un acuerdo muy inteligente, no sé si desde el punto de vista editorial, de cuyos negocios no entiendo mucho, pero sí desde el punto de vista de dejar oír una voz nueva, original y vigorosa [...]. Desde hace ya bastantes años, un grupo de amigos que conocimos los primeros cuentos y piezas teatrales de Juan Benet, le consideramos como uno de los escritores más dotados de nuestra generación, y siempre hemos esperado que alguien llegase a reconocerlo así. Me alegro de que sea precisamente usted, que siempre tuvo oído fino para la literatura, quien le abra la puerta de Áncora y Delfín [...]. (Martín Gaite y Benet, 2011: 151)

Los lectores de esta Correspondencia podrán comprobar que la carta a Vergés es una copia que Martín Gaite adjuntó a Benet dentro de otra misiva en la que se inventaba la broma de ser una empleada de la agencia literaria, La Rápida, S.A., a quien se le había asignado la encomienda de la pronta y esperada publicación de Volverás a Región. La chanza de esta carta firmada por el heterónimo Antonio Villarejo sirve para restar importancia al convencimiento de su mediación:

Adjunto le envío el borrador de la carta cuya redacción tuvo a bien encomendar a nuestra agencia literaria “La Rápida, S.A.”, trabajo del que se ha encargado personalmente la eximia y famosa escritora Carmen Martín Gaite. Suponemos que sabrá usted tener en cuenta la importancia de tal firma y ponderar el mérito de nuestra gestión. Se trata de una señora de

\footnotetext{
${ }^{1}$ Juan Benet ha relatado en La moviola de Eurípides cómo presentó su novela en 1965 al premio Nadal "y no alcanzó a clasificarse entre los veinte finalistas". Después intentó infructuosamente publicarla, hasta que Dionisio Ridruejo consiguió interesar “a su amigo personal José Vergés” (Benet, 1981: 32). Por otro lado, Martín Gaite, en su conferencia, "Juan Benet: La inspiración y el estilo", añade que "recién muerto Luis Martín-Santos [...], escritores jóvenes que tuvieran acceso a editoriales como Destino o Seix Barral, no conocía Juan a mediados de los sesenta más que a Ferlosio y a mí, que por cierto influimos mucho, junto con Dionisio Ridruejo, en la decisión de José Vergés para que Volverás a Región [...] fuera publicado en Destino” (Martín Gaite, 2017: 948-949). La novela se editó finalmente en febrero de 1968, tras superar imposiciones de la censura.
} 
difícil acceso y muy amargada por cuestiones sentimentales y financieras, pero al fin por medio de nuestro asalariado señor [Julián] Marías, ${ }^{2}$ que la ve todos los martes, la hemos podido convencer. (Martín Gaite y Benet, 2011: 150)

Este primer documento dirigido al director de Destino demuestra que Carmen Martín Gaite apoyó con sincera lealtad a Juan Benet en sus inicios de escritor, un dato de historia literaria que no conviene despreciar para evitar olvidos. Pese a las discrepancias entre las poéticas de ambos autores, desde sus primeras cartas Martín Gaite emitirá un juicio sobre el escritor Benet, que en el fondo siempre mantendrá: valora la altísima calidad de su prosa y la capacidad de forjar su propio estilo, pero también le advierte, desde 1964, de su tendencia a caer en lo "artificioso" y en extremar la dificultad (Martín Gaite y Benet, 2011: 43-44). Pero, para nuestro propósito, estas cartas nos interesan porque revelan cómo en esta labor de mediación editorial - ejercida como en la mayoría de los casos por su propia iniciativa- prevalecerán siempre los valores artísticos sobre los empresariales, a los que Martín Gaite decide hacer caso omiso. No podría ser de otra forma: opinaba la escritora por su propia convicción, desde su compromiso artístico, y por su real gana. Toda la labor de asesoramiento de Martín Gaite fue un recordatorio al editor de este deber ser: de la supremacía del valor artístico sobre el mercado. Desde luego - no es posible ponerlo en duda- esta mediación en favor de los criterios artísticos fue también una afirmación autoral. La misma autoridad como escritora que la llevará a reclamar en su correspondencia con la editora Esther Tusquets que difundiera mejor y con más eficacia sus obras publicadas en Lumen (Toribio Álvarez, 2018: 259-278).

La segunda secuencia de mi relato repara en una Carmen Martín Gaite madrina de los jóvenes editores (Mauricio d'Ors, Juan Antonio Molina Foix y Diego Lara) de Nostromo (1973-1976) (Teruel, 2015). Esta pequeña, pero pionera,

\footnotetext{
${ }^{2}$ Martín Gaite formó parte del Seminario de Estudios de Humanidades desde 1965 a 1969. La ayuda de la Fundación Ford a la Sociedad de Estudios y Publicaciones, vinculada al Banco Urquijo, permitió organizar este Seminario, dirigido por Julián Marías con la colaboración de Pedro Laín Entralgo, Enrique Lafuente Ferrari, Rafael Lapesa, José Luis Aranguren y Melchor Fernández Almagro. El Seminario se concentró en el estudio del siglo XVIII y el periodo romántico, como claves de comprensión de la España de 1960, y se reunía semanalmente en la llamada Casa de las Siete Chimeneas, de la plaza del Rey. Entre sus becarios, además de nuestra escritora, figuraron los nombres de Francisco Aguilar Piñal, Gonzalo Anes, Antonio Elorza, José Luis Cano, Elías Díaz, José María López Piñero, Miguel Martínez Cuadrado, Eduardo Martínez Pisón y María Cruz Seoane. Este Seminario significó para Martín Gaite la posibilidad de encauzar una vocación universitaria de investigadora, que aparcó a principios de los años cincuenta, cuando decidió "meterse a novelista" (Martín Gaite, 2016: 807).
} 
editorial se adelantó a muchas otras en el tardofranquismo y fue también motor del cambio mental que hizo posible la transformación del lector en la Transición, preparándole para leer literatura contemporánea en buenas traducciones. Nostromo tuvo posteriormente en Martín Gaite a su fiel biógrafa a través de artículos como "Réquiem por una editorial” y "Diego Lara” (Martín Gaite, 2016: 962-964; 974-975). No en vano "los nostromos" habían tomado como secretaria a su hija Marta Sánchez Martín, de dieciséis años.

“Mauricio d’Ors me pidió algún original mío para iniciar aquella colección, no sin antes advertirme que, de momento, me podrían pagar muy poco o nada", leemos en "Réquiem por una editorial" (Martín Gaite, 2016: 974). El primer número del catálogo de Nostromo fue su ensayo La búsqueda de interlocutor y otras búsquedas (cuya dedicatoria "Para Juan Benet, cuando no era famoso", engarza, a la altura de 1973, con toda la intencionalidad, con la carta de 1967 que acabamos de citar). Y en ese mismo catálogo se publicó también su primera traducción del portugués, El misterio de la carretera de Sintra (1974), de Eça de Queiroz y Ramalho Ortigão. Probablemente, en este madrinazgo, Martín Gaite tuvo en mente otro reducto de libertad de la que había disfrutado veinte años atrás; me refiero a aquella aventura juvenil, arriesgada y moderna que en la España del medio siglo significó Revista Española (1953-1954), en la que los jóvenes prosistas madrileños fueron apadrinados por el bibliófilo y entonces asesor de la editorial Castalia, Antonio Rodríguez Moñino. El papel protector y de mecenas de Martín Gaite revela también un rasgo distintivo de su ejecutoria de mediación editorial: su curiosidad por interesarse en la relación que los jóvenes mantenían con la literatura. La misma inclinación que la llevará, con entusiasmo y exigencia, a convertirse en un icono de la vida literaria madrileña en la década de 1990, como reconocerá agradecido Álvaro Pombo (2011: 46).

La correspondencia de Martín Gaite con Juan Benet y, sobre todo, con Gabriela Sánchez Ferlosio - algunas de estas cartas hasta ahora inéditas serán incluidas en el próximo y último volumen de sus Obras completas (2019) — da noticia lateral del único empleo, pero sin horario fijo, que conocemos en la biografía de nuestra escritora: un trabajo de media jornada en Salvat Editores, que se prolongó ocho meses, desde octubre de 1973 a junio de 1974, y que será el tercer hito de nuestro itinerario. "Se puede decir que he vivido exclusivamente de la pluma, como era mi deseo. [...] Siempre he evitado, aun a costa de vivir más modestamente, los empleos que pudieran esclavizarme y quitarme tiempo para dedicarme a la lectura, a la escritura y a otra de mis pasiones favoritas: el cultivo de la amistad", leemos en un esbozo autobiográfico escrito para lectores norteamericanos a petición de la hispanista Joan L. Brown (Martín Gaite, 2016: 651).

No sabemos con exactitud en qué consistió su trabajo en Salvat, solo constatamos por los documentos que acabamos de citar que escribía misivas entre 
"ciertos claritos que me dejaba mi labor de ejecutiva" (carta de 19 octubre de 1973 a Gabriela Sánchez Ferlosio [Martín Gaite, 2019; en prensa]); o que tomaba notas, desde su despacho mañanero de la madrileña calle del General Mola, en sus Cuadernos de todo (2002: 198), y que fue una tarea que desempeñó con poco entusiasmo, ya que interrumpía su verdadero oficio, como demuestra la misiva que vuelve a enviar a Gabriela Sánchez Ferlosio, el 1 de noviembre de 1973: "Son las ocho. Me voy a levantar, a hacerme un té, a ducharme, y a ponerme a escribir porque hoy es fiesta y no tengo oficina" (2019; en prensa). Martín Gaite estaba inmersa en la redacción de Retahílas (1974), la novela que supondrá el ansiado regreso a la ficción, tras su incursión en la investigación histórica con El proceso de Macanaz (1969).

No es imposible pensar que nuestra escritora asesorara a José Luis Alemán, editor responsable de Salvat en Madrid, sobre la selección de los últimos títulos, ediciones y prólogos de la colección Biblioteca Básica Salvat de Libros RTV —me lo sugiere también su amiga Milagro Laín, esposa de Alemán. Martín Gaite ya había prologado en esta colección El retrato de Dorian Gray (1970) y Los bravos (1971). Las cartas que conocemos emitidas desde Salvat Editores no ofrecen ninguna información sobre su cometido, básicamente justifican entre burlas y veras tan sorprendente decisión: "Parece que voy cumpliendo con acierto las misiones que se me encomiendan y que por ahora doy gusto a mis padres y a mis profesores", le comenta con sorna el 19 de octubre de 1973 a Gabriela Sánchez Ferlosio (2019; en prensa); "He cogido este trabajo de media jornada porque todo el invierno y la primavera pasados me aguantaba difícilmente a mí misma tantas horas en casa y Marta ya parando, en cambio, tan poco en ella”, explica a Juan Benet un mes más tarde desde su despacho (2011: 180). Más allá de las necesidades económicas y de su amistad con José Luis Alemán, quizá sea esta la razón última de su elección: hasta 1973 Martín Gaite tuvo que compatibilizar su labor de escritora e investigadora con educar y entretener a una hija en casa. ${ }^{3}$

El cuarto momento de este trayecto se detiene en 1978, año en el que Martín Gaite recibe el Premio Nacional de Narrativa por El cuarto de atrás y en el que formó parte del comité de lectura para autores extranjeros del proyecto Alfaguara de Jaime Salinas, con quien había trabado amistad desde mediados de 1960 en

\footnotetext{
${ }^{3}$ He mencionado en la secuencia anterior que Marta Sánchez Martín comenzó a trabajar precisamente en 1973, en la editorial Nostromo, “como 'secretaria-chica-para-todo' [...]. Le pagaban muy poco, pero era feliz. Aquel era su primer trabajo y ellos sus primeros amigos" (Martín Gaite, 2016: 975). Hasta entonces Marta se educó en el domicilio familiar bajo la tutela de sus padres y, en 1977, justo después del cierre de Nostromo, se matriculó en Filología Inglesa en la Universidad Complutense.
} 
Alianza Editorial, sello en el que la escritora publicó varias traducciones. ${ }^{4}$ Como sabemos, Salinas no solo cambió los objetivos de la editorial Alfaguara, sino también los procedimientos (Martínez Martín, 2015: 360), ya que fue partidario de delegar y de crear un comité de lectores, cuyas debilidades él conocía perfectamente, con vistas a que el contraste entre los distintos informes y poéticas le ayudase a tomar una decisión (Salinas, 2013: 38). Ese comité de lectura de autores en lengua no española estuvo formado en 1978 por Javier Marías, Juan Benet, Juan García Hortelano, Luis Goytisolo, Amaya Lacasa, Pablo Sorozábal y Carmen Martín Gaite (Salinas, 2013: 226). Se deriva fácilmente de estos nombres la diferencia entre sus distintas concepciones narrativas y, como consecuencia, la disparidad entre los autores y los títulos publicados en la colección Literatura Alfaguara en 1978: Thomas Bernhard (Trastorno), Patrick Modiano (Los bulevares periféricos), Günter Grass (El tambor de hojalata), Henry Miller (Trópico de Capricornio, Trópico de Cáncer y Sexus), Peter Handke (El miedo del portero al penalty), Gaetano Tumiati (El corsé de yeso), Nélida Piñon (Tebas de mi corazón) y Agustina BessaLuís (La sibila), entre otras traducciones.

Martín Gaite duró poco en este comité. La poética que se imponía no era de su completo gusto. Sus reseñas de algunos de estos títulos publicadas en Diario 16, entre 1978 y 1979, demuestran su rechazo a la exhibición del esplendor verbal y a las "triquiñuelas" faulknerianas de Trastorno, a la glacial frialdad de El miedo del portero al penalty, o a las pasajeras modas culturales de los best seller, como El tambor de hojalata. Su crítica a propósito de Poesía secular, de Ibn Gabirol, deja traslucir oblicuamente una opinión sobre la serie Literatura Alfaguara: "La colección Clásicos Alfaguara, la más acertada y rigurosa de cuantos componen esta casa editorial" (2017: 244). Aunque también es preciso reconocer que saludó con vivo entusiasmo Los bulevares periféricos de Patrick Modiano ${ }^{5}$ y La sibila de Agustina

\footnotetext{
${ }^{4}$ Entre 1968 y 1972 Martín Gaite tradujo para la colección El Libro de Bolsillo de Alianza: Vino y pan, de Ignazio Silone; Corto viaje sentimental, de Italo Svevo; Ocho siglos de poesía gallega. Antología bilingüe junto a Andrés Ruiz Tarazona; y Actitudes patriarcales: Las mujeres en la sociedad, de Eva Figes. Precisamente uno de los logros de Jaime Salinas como editor fue haber revalorado el papel y los derechos del traductor como autor. Por otro lado, la conciencia martingaitiana con el menester de la traducción queda evaluado en su artículo, "La ingrata condición del traductor. Bailar con la más fea” (2017: 209-210), publicado en Diario 16, el 24 de junio de 1978, coincidiendo con las fechas en las que formó parte del comité de lectura para autores extranjeros de Alfaguara.
}

\footnotetext{
${ }^{5}$ Uno de los rasgos distintivos de Martín Gaite como crítica literaria fue su manifestación efusiva del entusiasmo, que no se debe confundir con la ausencia de rigor, sino con una particular voz crítica necesitada de asideros plásticos y afectivos, que no encontraremos en otros críticos masculinos. En tal sentido comenta sin rubor de Los bulevares periféricos: "Patrick Modiano es de
} 
Bessa-Luís. Pero las razones de que su paso por este comité fuera breve no se explican solo desde motivos estéticos. Comenta Jaime Salinas:

Yo me resistía mucho a ver a Juan [Benet] cuando estaba con "los perros". ${ }^{6}$ Se volvía insoportable, montaba sus números de enfant terrible. Era su forma de tenerlos a raya. [...] Me molestaba no poder tener una conversación pausada, tranquila con Juan. En nuestra sociedad rara vez te encuentras reunido con gente que dialogue. Siempre hay alguien que toma el poder, domina, y la conversación se vuelve una lucha constante por convertirse en protagonista. (2013: 228-229)

Es muy probable que Carmen Martín Gaite suscribiera esta opinión de Salinas. De hecho, en su agenda inédita de 1978, el 31 de mayo, leemos esta escueta anotación: "En el show de Alfaguara". Martín Gaite no soportaba el esnobismo histriónico de ciertos espectáculos y sobresfuerzos por no parecer vulgar y huyó del aspaviento y del pavoneo intelectual. Entendió que la verdad artística era una representación compartida y concibió la literatura como todo lo contrario al discurso de los locos o los vanidosos. Por tanto, su vinculación con el comité de lectura de Alfaguara para autores en lengua extranjera fue inevitablemente corto, tanto por razones estéticas como extra estéticas. Se trata de otra de las recurrentes y discretas negativas de la Martín Gaite, a lo largo de su trayectoria intelectual (Gopegui, 2000: 37).

Tras la compra por Planeta de la mitad de Ediciones Destino en 1989, Carmen Martín Gaite decidió en ese mismo mes de julio entregar su obra futura a Jacobo Siruela y, sobre todo, a Jorge Herralde, con quien ya había publicado, dos años

esos escritores que se te meten por el alma" (2017: 207). "El libro resulta [...] cálido y acogedor en su misma ligereza, abriga sin pesar, como las mantas buenas" (2017: 142), es el expresivo símil que se le ocurre para los Relatos sobre la falta de sustancia, de Álvaro Pombo. "Uno de los cuentos de aventuras más hermosos que he leído en mi vida. ¡Cuánto le habría gustado a Ignacio Aldecoa!" (2017: 186), exclama recordando al amigo desaparecido tras la lectura de El silencio blanco, de Jack London. "Compré inmediatamente el libro y he pasado tres días memorables engolfada en su lectura" (2017: 318), así nos confiesa los efectos narcóticos destilados por la buena narración de Los hechos del rey Arturo y sus nobles caballeros. "Quiero cerrar estas divagaciones dando un consejo de aficionada, el único que se me ocurre para reivindicar la libertad en un reino tan abocado al naufragio como es el de la literatura: por mucho que os insten a ello los entendidos, no leáis nunca una novela que os aburra", termina recomendando en "Morir aprendiendo" (2017: 112). Son algunos ejemplos de los atributos diferenciadores de su crítica literaria: su defensa de la afición y su disposición para convertir el análisis literario en un momento de su autobiografía espiritual.

6 "Los perros" fue el apelativo que recibieron los jóvenes Javier Marías, Vicente Molina Foix y Félix de Azúa. A "los perros" por contestatarios había que ponerles "el bozal y la cadena" en cuanto entraban, comenta Jaime Salinas (2013: 228). 
antes, Usos amorosos de la postguerra española (Xv premio Anagrama de Ensayo), que pese a ser, en parte, un libro de investigación histórica se convirtió en lo que su editor llamó cuidadosamente un long-seller (Herralde, 2001: 60). En espera de la apertura del Archivo de Anagrama prevista para 2019, la memoria de la editorial está depositada en el anecdotario que ha difundido oralmente y por escrito Jorge Herralde. Tanto de Opiniones mohicanas como de Por orden alfabético es posible extraer evidencias del papel que Carmen Martín Gaite jugó en la década de 1990 como cónsul anagramática en Madrid para la joven narrativa. Este será el último momento en el relato trazado de la intercesión editorial de Martín Gaite, pero reviste particular significación, ya que su papel de mediadora de lo estético, de los valores literarios, servirá claramente para la toma de decisiones empresariales de su editor. Martín Gaite venía avalada por el marchamo de que Nubosidad variable fue "la novela de mayor éxito de la colección 'Narrativas hispánicas", como reconoció el propio Herralde (2001: 59). Por tanto, este papel de mediación es precedido por su autoridad de escritora, que termina incrementándose a su vez. Así lo vimos en su apoyo a Benet en 1967, pero sin duda fue aquel un episodio que quedó aislado y eclipsado por el posterior ascendente del autor de Volverás a Región entre la generación novísima.

"Quien primero me habló de Chirbes fue Carmen Martín Gaite [...]. Ella me pasó el manuscrito de una novela, Mimoun" y "fue nuestra persona en Madrid para varios jóvenes escritores”, recuerda Jorge Herralde $(2006: 78,251)$. Estos jóvenes principiantes fueron Rafael Chirbes, Álvaro Pombo, Miguel Sánchez-Ostiz y Belén Gopegui. Su papel de asesoramiento voluntario se completó con la función de madrinazgo a través de la crítica literaria que ejerció semanalmente desde 1976 en Diario 16 y posteriormente en otros medios, como El Sol o Saber Leer, donde reseñó los primeros títulos de Juan José Millás, Marina Mayoral, Rosa Montero, Soledad Puértolas, Esther Tusquets, Manuel Longares, Eloy Tizón y Antonio Muñoz Molina. Carmen Martín Gaite fue especialmente generosa con los narradores noveles, a quienes otorgó siempre un voto de confianza. Aunque también es preciso remarcar el estímulo y el acicate de modernidad que supuso para ella y su obra el trato con los más jóvenes - tanto en vida como después de la muerte de su hija Marta. Su producción literaria manifiesta además una fuerte sensibilización con los conflictos intergeneracionales, como se desprende de El cuarto de atrás (1978), Usos amorosos de la postguerra española (1987), Esperando el porvenir (1994), Visión de Nueva York (2005) y su último ciclo narrativo, desde Nubosidad variable (1992) a Los parentescos (2001).

De este relato en cinco hitos de la escritora como mediadora editorial se desprenden dos cuestiones centrales. La primera atañe a la pertinencia de contar también con las mediadoras, asesoras y agentes literarias a la hora de estudiar el protagonismo femenino en la edición contemporánea. La segunda afecta 
directamente a la biografía intelectual de Carmen Martín Gaite. Conocíamos la variedad de sus intereses intelectuales y cómo se desplegaron en múltiples direcciones, desde los géneros literarios consabidos (cuento, poesía, novela, teatro y ensayo) a ese híbrido llamado "cuaderno de todo", desde la investigación histórica al artículo periodístico, desde la traducción a las adaptaciones teatrales de los clásicos y los guiones para televisión. Su trayectoria es un paradigma de mujer de letras en la cultura española del siglo Xx y ese papel se refuerza con esta labor que hemos comenzado a documentar de asesoramiento y madrinazgo editorial a la que dedicó su "afición" lectora y que potenció al mismo tiempo su auctoritas. La vida y obra de Martín Gaite están presididas por la curiosidad intelectual y por la búsqueda de un lugar desde el que afrontar con dignidad la tarea de vivir en una España donde la grisura franquista había sido sustituida por la falta de sustancia, tras una Transición llamada democrática (Chirbes, 2011: 3). Para ella la creación literaria, la crítica y la mediación editorial fueron actos de una misma función: su compromiso con la literatura, que la llevó a apoyar a la joven narrativa siempre que traspasase el umbral de su exigencia. Sin duda, fue "un aspecto no demasiado frecuente entre los escritores consagrados" (Herralde, 2001: 57).

\section{REFERENCIAS BIBLIOGRÁFICAS}

Benet, Juan (1981), La moviola de Eurípides, Madrid, Taurus.

Chirbes, Rafael (2011), "Puntos de fuga”, El legado de Carmen Martín Gaite, José Teruel (coord.), Ínsula: Revista de letras y ciencias humanas, enero-febrero, 769-770: 2-4.

Gopegui, Belén (2000), “El sí de cada no”, El País, 24 de julio: 37.

Herralde, Jorge (2001), Opiniones mohicanas, Barcelona, El Acantilado.

-(2006), Por orden alfabético. Escritores, editores, amigos, Barcelona, Anagrama.

Martín Gaite, Carmen (2002), Cuadernos de todo, Maria Vittoria Calvi (ed.), Barcelona, Debate/Círculo de Lectores.

-(2016), Obras completas V. Ensayos II. Ensayos literarios, José Teruel (ed.), Barcelona/Madrid, Círculo de Lectores/Espasa.

-(2017), Obras completas VI. Ensayos III. Artículos, conferencias y ensayos breves, José Teruel (ed.), Barcelona/Madrid, Círculo de Lectores/Espasa.

-(2019), Obras completas VII. Cuadernos y cartas, José Teruel (ed.), Barcelona/Madrid, Círculo de Lectores/Espasa. [en prensa]

Martín Gaite, Carmen y Juan Benet (2011), Correspondencia, José Teruel (ed.), Barcelona, Galaxia Gutenberg/Círculo de Lectores. 
Martínez Martín, Jesús A. (2015), “La transición editorial. Los años setenta”, Historia de la edición en España (1939-1975), Jesús A. Martínez Martín (dir.), Madrid, Marcial Pons: 328-386.

Pombo, Álvaro (2011), "Una aguja en un pajar", Ínsula: El legado de Carmen Martín Gaite, José Teruel (coord.), enero-febrero, 769-770: 46.

Salinas, Jaime (2013), El oficio de editor. Una conversación con Juan Cruz, Madrid, Alfaguara.

Teruel, José (2015), “Editorial Nostromo (1973-1976) [Semblanza]", portal Editores y Editoriales Iberoamericanos (siglos XIX-XXI), EDI-RED, Alicante, Biblioteca Virtual Miguel de Cervantes. <http://www.cervantesvirtual.com /obra/editorial-nostromo-1973-1976-semblanza/>

Toribio Álvarez, Andrea (2018), "Historia de una correspondencia: Carmen Martín Gaite y Esther Tusquets", Historia e intimidad. Epistolarios y autobiografía en la cultura española del medio siglo, José Teruel (ed.), Madrid y Frankfurt, Iberoamericana-Vervuert: 259-278. 